ISSN 1112-9867

http://www.jfas.info

\title{
EFFECT OF URBAN ALBEDO SURFACES ON THERMAL COMFORT
}

\author{
O. Mansouri ${ }^{1,2, *}$, F. Bourbia ${ }^{1}$ and R. Belarbi ${ }^{2}$ \\ ${ }^{1}$ Laboratoire ABE, Université de Constantine3, Ville Nouvelle Ali Mendjeli, Constanine \\ 25000 Algérie \\ ${ }^{2}$ Laboratoire LaSIE, Université de La Rochelle, Avenue Michel Crépeau 17042. La Rochelle. \\ France
}

Received: 15 October 2016 / Accepted: 16 April 2017 / Published online: 01 May 2017

\begin{abstract}
Thermal comfort was and remains a major concern of the designers, makers, planners, climatologists and others, seeking at all costs to ensure the well-being, good health and comfort of citizens in both outdoor and indoor areas. They have addressed this issue in order to identify the main causes that generate the warming of urban areas and therefore contribute to the degradation of the exterior and interior thermal comfort of the inhabitants. It turns out that the reflectivity of materials known as the albedo, plays a leading role in this degradation. A numerical study was carried out to assess the thermal comfort of citizens by applying reflective materials for roofs, facades and floor. The results show that the reflective light paints help in moderating the microclimate, contrariwise, they participate in the degradation of thermal comfort.
\end{abstract}

Keywords: Reflectivity; Materials; Envi-met; Rayman; Degradation.

Author Correspondence, e-mail: wmansouri_2006@yahoo.fr

doi: http://dx.doi.org/10.4314/jfas.v9i2.17

\section{INTRODUCTION}

Les effets du réchauffement climatique et la hausse des temperatures dans les centres urbains ont pris aujourd'hui des proportions qui inquiètent les scientifiques, en particulier les 
climatologues. Ceux-ci divulguent les impacts d'un réchauffement qui menace les grands équilibres planétaires, et précisent que les systèmes urbains en sont les grands responsables dans la mesure où ils représentent plus de $75 \%$ de la consommation globale de l'énergie [1]. La manifestation la plus connue du microclimat généré par les villes suite à cette hausse de temperature est l'îlot de chaleur urbain "ICU”, qui peut varier de $2^{\circ} \mathrm{C}$ pour les petites villes de quelques milliers d'habitants à $12^{\circ} \mathrm{C}$ pour des villes de plusieurs millions d'habitants [2]. Les chercheurs annoncent que le changement des propriétés de la surface - impérméabilité des revêtements, matériaux de grande capacité thermique et retrait de la végétation créant un environnement propice au piégeage du rayonnement solaire -, influent sur la modification du climat en ville plus que la source additionnelle de chaleur dégagée par les activités humaines [2]. De ce fait, l'émergence du réchauffement climatique et les nouveaux risques à la fois écologiques et sanitaires qu'il provoque, ont necessité une démarche qui prends au sérieux les réflexions sur le phenomène d'ICU.

\section{ETAT DE L'ART}

Les matériaux urbains se caractérisent fréquemment par l'importance de leur capacité calorifique et de leur conductivité thermique. L'énergie est emmagasinée durant la journée et restituée peu à peu par rayonnement au cours de la nuit. C'est ainsi que le refroidissement nocturne est plus lent en ville que dans des zones non urbanisées. En fait, l'une des strategies visant à combattre l'ICU consiste précisément à tenter de modifier l'albédo. Taha et al. [3], par exemple, en 1988, ont trouvé que l'augmentation de l'albédo des surfaces jumelée avec d'autres procédés tel que l'augmentation de la couverture végétale, joue un rôle primordial dans l'atténuation de l'îlot de chaleur urbain, et réduit le coût de conditionnement de l'air. Ils ont simulé la reduction de la charge de refroidissement d'un bâtiment de 18,9\% pour les journées d'été à Sacramento, en Californie, pour une augmentation de l'albédo des toits et des murs de 0,30 à 0,90. Les simulations indiquent que le badigeonnage (blanchissement) du bâtiment produit des gains directs de $14 \%$ et $19 \%$ sur le pic de puissance de refroidisssement et sur l'énergie électrique de refroidissement respectivement. La modification à la fois de l'albédo urbain et du badigeonnage du bâtiment, peut entrainer une économie totale de 35\% et 
de $62 \%$ respectivement. Une autre recherche de Taha et al. en 1992 [4], a montré que l'utilisation des matériaux d'albédo élevé réduit la quantité de rayonnement solaire absorbé par l'enveloppe des bâtiments et des structures urbaines et maintient leurs surfaces fraîches. Ils ont mesuré l'albédo et les températures de surface d’une variété de surfaces. Ils ont constaté, par exemple, que les revêtements élastomères blancs (avec un albédo de 0,72) étaient de $45^{\circ} \mathrm{C}$ plus froids que les revêtements noirs (avec un albédo de 0,08 ) en début d'après-midi d'un jour clair en été. Une surface blanche avec un albédo de 0,61 était seulement $5^{\circ} \mathrm{C}$ plus chaude que l'air ambiant alors que le gravier conventionnel avec un albédo de 0,09 était de $30^{\circ} \mathrm{C}$ plus chaud que l'air. Taha [5] rapporte aussi suite à des simulations tridimensionnelles méso-échelle sur les effets d'une large échelle d'albédo croissant dans le bassin de Los Angeles qu'une moyenne de $2^{\circ} \mathrm{C}$ jusqu'à $4^{\circ} \mathrm{C}$ peut être possible par l'augmentation de l'albédo par 0.13 dans les zones urbanisées du bassin. Rosenfeld et al. [6] ont démontré qu'en augmentant l'albédo des surfaces urbaines et en plantant des arbres, le phénomène de l'ICU est atténué. Une grande augmentation de l'albédo, combiné avec l'ombrage des arbres peut réduire de $40 \%$ l'usage de l'énergie pour la climatisation. De leur part, Chatzidimitriou et al.2006 [7], ont mené une étude sur plusieurs matériaux couvrant les surfaces urbaines telles que le marbre, la pierre, le béton, les tuiles en céramique et l'asphalte aussi bien que l'eau et l'herbe. En effet, les mesures effectuées durant les conditions chaudes d'été, afin d'évaluer l'effet des differents matériaux sur le microclimat, révèlent un rapport entre les températures de surface et l'albédo des surfaces, où les températures les plus basses ont été observées avec les plus hauts albédo excepté le cas de l'herbe où l'influence de l'évapotranspiration, l'ombre et la capacité thermique du sol était plus grande. Les températures de l'air semblent avoir une corrélation avec les températures de surface, excepté pour le marbre blanc où la température moyenne de l'air la plus élevée a été enregistrée (jusqu'à 6.6\% plus élevée que celle au dessus du marbre noir) en dépit de sa surface plus froide. Cela peut être attribué d'après les auteurs à la haute réflectivité de la surface blanche et la quantité importante du rayonnement solaire réfléchi qui a probablement affecté les capteurs.

D'autres études, menées par Shiraishi et al. [8] signalent que les températures moyennes radiantes les plus hautes sont dans les zones avec un haut albédo, comparées à des surfaces 
réflectives moindres. Santamouris [9], quant à lui, rapporte en 2006 qu'après le développement d'une nouvelle génération de peintures fraîches qui présente une plus haute réflectivité du spectre solaire parmi toute les peintures existantes et garantie le maximum de bénéfices thermiques pour le bâtiment dans les climats chauds, des mesures extensives effectuées ont prouvé que la réflectivité solaire de la nouvelle génération des peintures fraîches excédait les 95\%. L'auteur a effectué une étude comparative des températures de surface pour diverses peintures extérieures, exécutées durant une saison estivale entière, il a prouvé que la nouvelle génération des peintures fraîches présentait durant le jour des températures plus basses d'environ $6^{\circ} \mathrm{C}$ que les marbres blancs. Akbari et al. [10] ont surveillé le pic de puissance et les économies d'énergie de refroidissement pour des revêtements à haut albédo d'une maison et deux bungalows scolaires à Sacramento, en Californie. Ils ont trouvé des économies de $2.2 \mathrm{kwh} / \mathrm{j}$ pour une maison ( $80 \%$ des cas d'utilisation de base), et la réduction de la demande de pointe de 0,6kw. Dans les bungalows de l'école, l'énergie de refroidissement a été réduite de $3.1 \mathrm{kwh} / \mathrm{j}$ (35\% des cas d'utilisation de base), et la demande de pointe par 0.6kw. Prado et Ferreira en 2005 [11] ont mené une étude par l'utilisation d'une variété de matériaux pour les toitures des constructions au Brésil, afin d'aboutir à des solutions passives qui peuvent atténuer les effets négatifs des îlots de chaleur urbains. Ils ont déduit que les céramiques blanches et rouges sont les seuls matériaux parmi plusieurs mesurés qui avaient atteint des températures de surfaces plus basses que les températures de l'air. Par contre, les matériaux métalliques qui ont des performances similaires à celles des céramiques blanches et rouges, avec des valeurs d'albédo d'environ 55\% ont montré des températures de surface beaucoup plus hautes, cela est dû à la basse émissivité des matériaux métalliques sans peinture qui émettent peu de radiations thermiques.

\section{DESCRIPTION DE LA METHODOLOGIE}

La présente étude s'est déroulée dans le quartier des 150 logements sociaux participatifs donnant sur le boulevard Houari Boumèdiene, au sud de Skikda, ville du Nord-Est de l'Algérie (latitude $36.54^{\circ} \mathrm{N}$ et longitude $6,52^{\circ} \mathrm{E}$ ), c'est une ville cotière caractérisée par le climat méditérranéen (chaud et humide en été et doux, pluvieux, humide, avec de faibles 
amplitudes en hiver). La température moyenne est de l'ordre de $26.09^{\circ} \mathrm{C}$ pour le mois d'août qui est le mois le plus chaud et de l'ordre de $12.07^{\circ} \mathrm{C}$ pour le mois de février qui est le mois le plus froid de l'année. Les humidités relatives moyennes mensuelles varient entre $67.1 \%$ et $70.83 \%$, ce qui explique que le climat de la ville est humide pendant toute l'année. Le rayonnement solaire est intense, la durée d'insolation moyenne mensuelle marque un total de 2669heures avec une énergie incidente maximale sur un plan horizontal de $8010 \mathrm{w} / \mathrm{m}^{2}$ pendant le mois de juillet [12]. La ville de Skikda est bien arrosée avec un total annuel des precipitations de $825.82 \mathrm{~mm}$ et une valeur maximale de $130.22 \mathrm{~mm}$ en décemble. Pour les vents, ils sont généralement faibles avec une moyenne annuelle de $3.43 \mathrm{~m} / \mathrm{s}$ de direction Nord-Est et Sud-Ouest en été, et Nord-Ouest à Nord-Est en saison froide, en plus de l'alternance des brises de terre et brises de mer [13].

Le cas d'étude est un espace extérieur entre deux blocs de bâtiments, qui joue le rôle d'aire de jeu pour les enfants, de lieu de rencontre pour les adultes ainsi qu'une place de parking pour les habitants. La Fig.1 montre une vue sur l'espace étudié insérré dans son site.

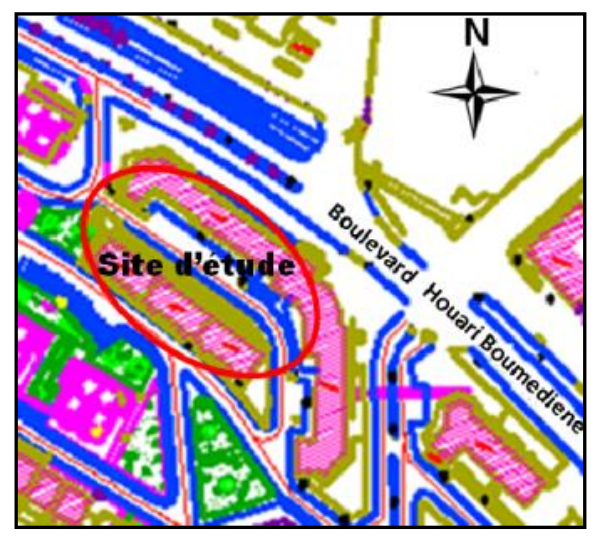

Fig.1. Présentation du cas d'études dans le site choisi

Source: POS S.Boulkeroua (DUC Skikda, 2014)

Afin d'estimer l'effet de l'albédo sur les conditions de confort thermique au niveau de l'espace choisi, nous avons effectué des simulations par le biais de deux logiciels, le modèle numérique microclimatique pronostique tridimensionnel Envi-met 3.1, pour déduire les différents paramètres microclimatiques (températures surfaciques du sol, températures de l'air, Température moyenne radiante), mais surtout pour évaluer le confort thermique extérieur dans l'aire d'étude par le calcul de l'indice du vote moyen prévisible PMV (Predicted Mean Vote), 
et le modèle numérique Rayman 1.2 pour calculer un autre indice de confort qui est la température physiologique équivalente PET (Physiological Equivalent Temperature).

La journée type choisie pour accomplir les simulations est le 28 juin 2014, c'est une journée chaude d'été reflétant la période de surchauffe avec des températures élevées marquant une valeur maximale de $34.1^{\circ} \mathrm{C}$ à $12 \mathrm{~h}$, un vent faible fluctuant de 0 à $3 \mathrm{~m} / \mathrm{s}$ et un ciel tout à fait dégagé.

Nous avons débuté les simulations en prenant en premier lieu les peintures existantes appliquées sur les différentes surfaces urbaines du site, (murs peints en couleurs blanche et bleue, toiture peinte en couleur grise, chaussée et trottoir peints en noir et gris); toute ces couleurs sont ternes, fades, ayant perdu leur éclat avec le temps avec l'existence d'une couche de poussière qui accentue d'avantage leur perte d'intensité et de vivacité, puis en deuxième lieu, en attribuant des couleurs réfléchissantes dites «cool» ou «froides» pour ces différentes surfaces, afin de pouvoir estimer l'effet de la réflectivité des matériaux sur l'ambiance thermique extérieure. Les valeurs attribuées à ces surfaces sont des valeurs empiriques déduites à partir de recherches précédentes dans le domaine de la climatologie urbaine et l'environnement à travers le monde.

Les valeurs initiales (réelles) d'albédo utilisées pour la simulation sont : 0.3 pour les murs, 0.15 pour l'asphalte de la chaussée, et 0.2 pour la toiture. Les valeurs attribuées à ces surfaces et qui représenteront des peintures réfléchissantes «cool» sont les suivantes: 0.75 pour les murs, en se référant aux résultats de recherche de Reagan et Acklam (1979) [14], qui ont suggéré des couleurs réfléchissantes pour les matériaux opaques de bâtiment. Une valeur de 0.51 pour l'asphalte de la chaussée, suite à la recherche de M.Pomerantz, et H.Akbari à Los Angeles en Californie ( 2005) [15] , qui ont développé un prototype d'asphalte d'une valeur de 0.51 qui a permis d'avoir une réduction de température de $15^{\circ} \mathrm{C}$ comparé à un asphalte clair et âgé d'une valeur de 0.15 . Nous avons changé uniquement la valeur d'albédo de la chaussée faite en asphalte, par contre, pour les parties du sol couvertes en pavage de béton et de la terre, elles n'ont pas été changées pour les deux cas de simulation (car leur surfaces étaient minimes d'une part, et d'autre part on a voulu voir l'effet net de l'asphalte de la chaussée sur les températures). Et enfin pour la toiture, on a attribué une valeur d'albédo de 
0.65, tirée des résultats de recherche de Synnefa, Santamouris et Akbari (2007) [16].

Le fichier édit d'Envi-met est représenté par la Fig.2 , c'est un espace dièdre orienté NO-SE d'un SVF (Sky View Factor ou facteur d'ouverture au ciel) de 0.51.

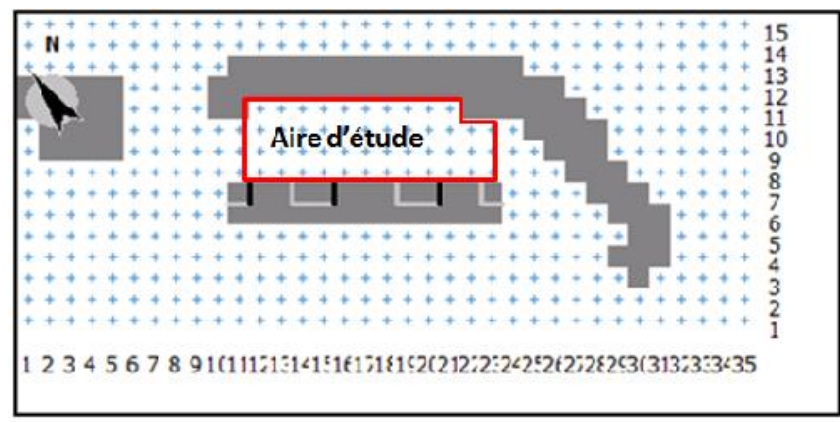

Fig.2. Fichier éditeur (.in) du cas d'étude par Envi-Met

\section{ANAL YSE DES RESULTATS}

\subsection{Paramètres climatiques}

\subsection{Températures de surface du sol}

Les résultats des simulations sont lus entre $6.00 \mathrm{~h}$ du matin et $20.00 \mathrm{~h}$, période d'exploitation de l'espace par les usagers. Le graphe de la variation de la température surfacique du sol représenté par la Fig.3 montre clairement qu'il y a une différence entre les peintures standards et les peintures réfléchissantes aux moments où le rayonnement solaire est le plus intense, en constatant une relation inversement proportionnelle entre la valeur d'albédo et celle de surface du sol, c'est entre 10h et $19 \mathrm{~h}$ qu'on remarque que les courbes des températures surfaciques relatives aux deux peintures s'éloignent, et plus précisément entre $11 \mathrm{~h}$ et $16 \mathrm{~h}$ en enregistrant un écart maximal de $6.43^{\circ} \mathrm{C}$ à $14 \mathrm{~h}$, car le rayonnement solaire pendant ces heures atteint son maximum et la fraction réfléchie est maximale, ce qui maintient les surfaces réfléchissantes fraîches comparées aux surfaces standards, et après 14h qui marque l'apogée des deux courbes, les variations relatives aux deux peintures maintiennent le même ordre, mais dans le sens décroissant en enregistrent une baisse des températures jusqu'à 19h. 


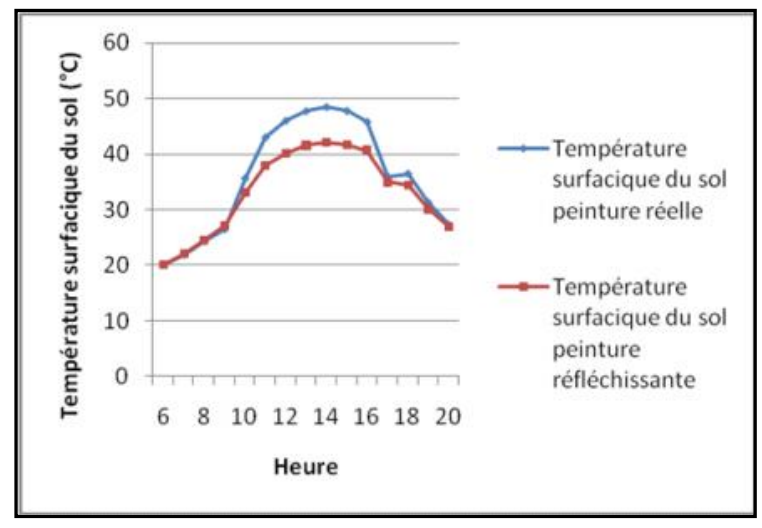

Fig.3. Variation de la température de surface du sol pour les peintures réelles (standard) et les peintures réfléchissantes (cool)

Ces résultats coïncident avec ceux de plusieurs recherches précédentes, parmi lesquelles celle de Chatzimitriou et al. en 2006, en Grèce [7], qui ont évalué l'effet des différents types de matériaux couvrant le sol des espaces urbains sur le microclimat pendant la période estivale. Les mesures effectuées ont révélé qu'il y a une relation inversement proportionnelle entre les températures de surfaces et l'albédo, c'est-à-dire que les basses températures de surfaces étaient observées sur les surfaces de matériaux ayant un haut albédo. Santamouris et al. en 2004 [17], de leur part, ont trouvé que les températures moyennes journalières de surfaces des carreaux en marbre blanc étaient de $29.7^{\circ} \mathrm{C}$ et celles des carreaux en asphalte étaient de $46.7^{\circ} \mathrm{C}$, à savoir que l'albédo du marbre blanc est plus élevé que celui de l'asphalte.

\subsection{Températures de l'air}

D'après le graphe relatif à la variation des températures de l'air pour des peintures réelles (standards) et d'autres réfléchissantes (cool), représenté par la Fig.4, on remarque que les deux courbes sont pratiquement confondues, mis à part quelques infimes différences en milieu de journée où la peinture réelle (standard) marque des valeurs légèrement plus élevées que celles de la peinture réfléchissante avec un écart qui atteint $0.23^{\circ} \mathrm{C}$ à $14 \mathrm{~h}$.

Ce qui s'explique par l'échauffement de l'air par convection car la vitesse du vent est faible. Donc la réflectivité des matériaux contribue à l'adoucissement et la modération du microclimat. 


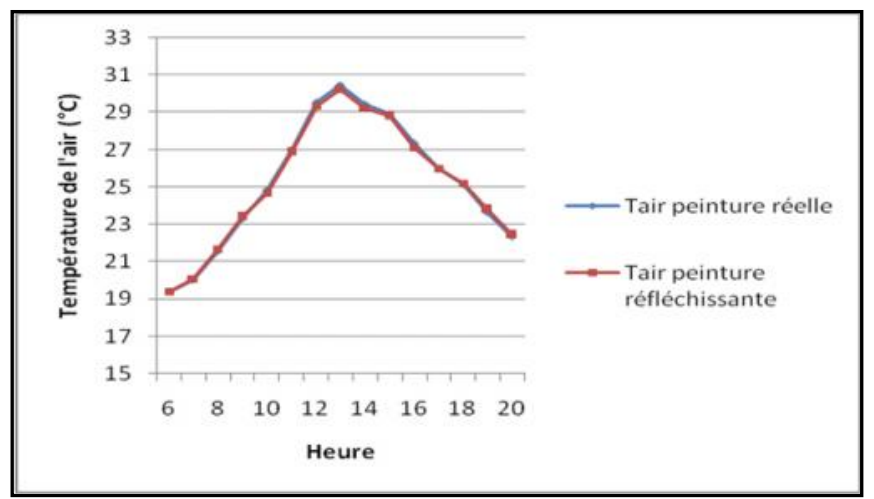

Fig.4. Variation de la température de l'air pour les peintures réelles et les peintures réfléchissantes

Cela a été constaté par R.Priyadarsini et N.H.Wong (2005) à Singapour [18], qui ont trouvé que pour un canyon étroit (qui représente un espace extérieur) avec une faible vitesse de vent, la température de l'air pourra augmenter de $2.5^{\circ} \mathrm{C}$ avec des matériaux de façade ayant une plus basse réflectivité, quoi que la différence pour notre cas est presque négligeable et cela est dû probablement à la géométrie de l'espace, surtout son SVF qui est égal à 0.51 .

\subsection{Températures moyenne radiante}

La température moyenne radiante «Tmrt» est la somme des températures des surfaces qui entourent le corps humain. La Tmrt est directement proportionnelle avec l'albédo des matériaux, c.-à-d. qu'elle augmente en fonction de l'augmentation de la réflectivité, elle est définie comme étant «la température d'une enceinte imaginaire uniforme dans laquelle le transfert de chaleur par rayonnement du corps humain est égal au transfert de chaleur par rayonnement dans l'enceinte réelle non uniforme » (ISO 7726, 1998) [19].

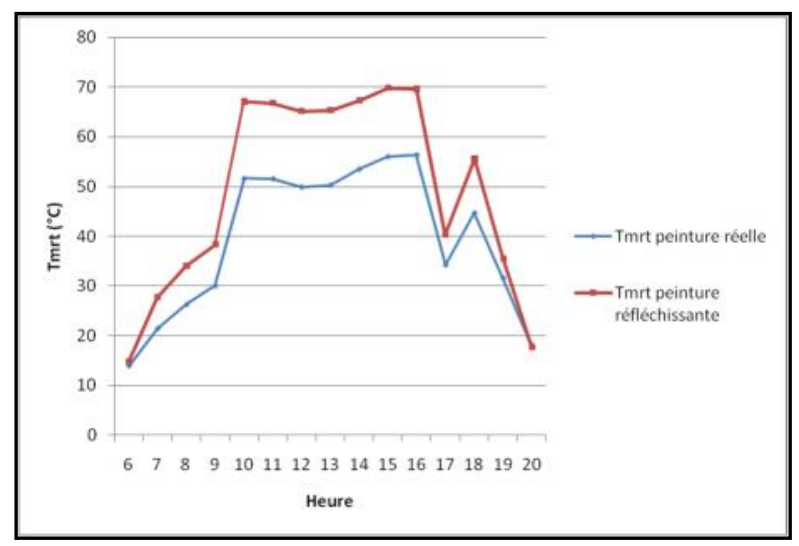

Fig.5. Variation de la température moyenne radiante pour les peintures réelles et les peintures réfléchissantes 
Des études récentes indiquent que la Tmrt a une forte corrélation avec l'équilibre énergétique humain et le confort thermique, elle est souvent un paramètre plus fiable que la température de l'air pour évaluer le confort thermique (F.Ali-Toudert et H.Mayer 2006[20], Halawa et al.2014 [21]).

Il est clair d'après le graphe que la Tmrt relative à la peinture réfléchissante marque des valeurs plus élevées que celles de la peinture standard, de 6h du matin jusqu'à 19h, en enregistrant un écart maximal de $15.47^{\circ} \mathrm{C}$ entre $10 \mathrm{~h}$ et $13 \mathrm{~h}$, cela est du à l'intensité du rayonnement solaire réfléchi (pendant les heures de fort ensoleillement) provenant des différentes surfaces urbaines -façades et chaussées-. Ces résultats peuvent s'aligner à ceux trouvés par Y.Shiraishi et al., (2005) au Japon [8], qui signalent que les températures moyennes radiantes les plus hautes sont dans les zones avec un haut albédo comparé à des surfaces réflectives moindres.

Cela coïncide aussi avec les résultats d'une recherche menée aux Pays-Bas, par Taleghani et al.en 2014 [22], qui disent que la température moyenne radiante à l'intérieur des cours avec des façades à haut albédo pendant l'exposition solaire est plus élevée que dans les cours avec des façades à faible albédo. A cause de l'albédo plus élevé, le rayonnement solaire est réfléchi encore plus en direction du point central du récepteur. Puisque une cour est une forme urbaine fermée, il ya une probabilité plus faible pour dissiper les reflets solaires provenant des bâtiments.

Dans une autre étude toujours de Taleghani et al. (2014) [23], les auteurs ont montré que dans le climat tempéré de Portland (OR, USA), une mesure sur une matière blanche (avec albédo $0,91)$ a augmenté la température globe et la température moyenne radiante de $\left(0,9^{\circ} \mathrm{C}\right.$ et $2,9^{\circ} \mathrm{C}$ respectivement), tout en produisant une température plus fraîche de l'air local $\left(1,3^{\circ} \mathrm{C}\right)$ en comparaison avec un revêtement noir (avec albédo 0,37). Pour résumer, l'effet de l'albédo à la fois sur l'intérieur et sur l'environnement thermique extérieur peut être déterminé selon les auteurs seulement lorsqu'il est étudié plus en détail pour chaque situation urbaine.

Les chercheurs Ghaffarianhoseini et al. De leur part dans une étude récente en 2015 [24], qui ont développé trois scénarios sur la base de l'augmentation de l'albédo des murs d'enceintes: les valeurs d'albédo sont de : 0,3 (brique), 0,55 (marbre) et 0,93 (plâtre hautement 
réfléchissant). Les résultats obtenus avec différentes valeurs d'albédo montrent que de $7 \mathrm{~h}$ à 20h, une cour avec un albédo plus élevé a une température moyenne radiante considérablement plus élevée. Ils affirment que la Tmrt dans la cour avec un albédo élevé de 0,93 est de $12^{\circ} \mathrm{C}$ supérieur à $12 \mathrm{~h}$ par rapport à celle de la cour avec l'albédo de 0,3

\subsection{Rayonnement réfléchi}

$\mathrm{Au}$ premier abord, les valeurs du rayonnement réfléchi pour les peintures réfléchissantes sont nettement beaucoup plus élevées que les valeurs du rayonnement pour les peintures réelles, notamment et spécifiquement pour les heures d'ensoleillement de la journée, avec un écart maximal de $155.02 \mathrm{w} / \mathrm{m}^{2}$ à $12 \mathrm{~h}$. Cela s'explique par le fait que le rayonnement réfléchi a une relation avec la surface d'exposition, c'est-à-dire que les surfaces avec des couleurs claires "froides" réfléchissent beaucoup plus le rayonnement que celles avec des couleurs plus sombres.

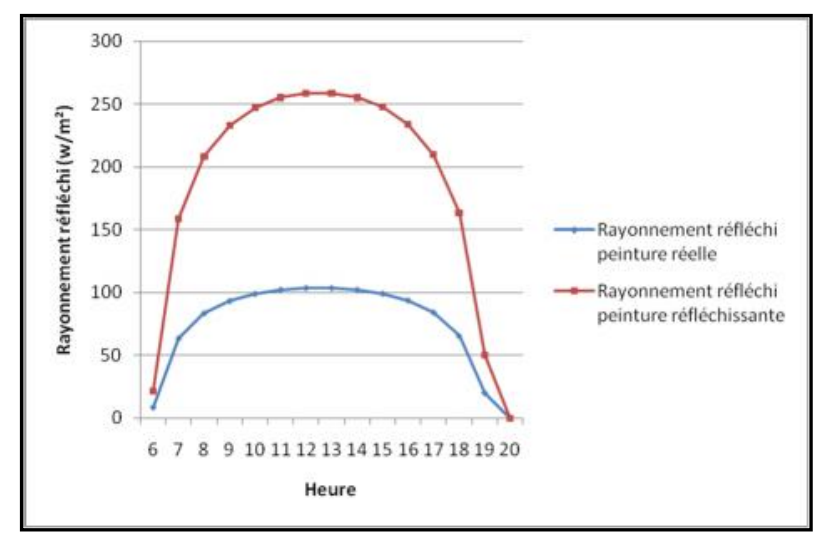

Fig.6. Variation du rayonnement réfléchi pour les peintures réelles et les peintures réfléchissantes

\subsection{Paramètres de confort thermique}

\subsection{Vote Moyen Prévisible}

Le PMV est Le vote moyen prévisible (Predicted Mean Vote), il a été défini par Fanger en 1972 [25]. Il relie le simple équilibre d'énergie pour l'échange de chaleur entre le corps humain et l'environnement thermique.

Fanger est arrivé à l'évaluation moyenne de l'environnement thermique par le PMV pour un grand échantillon d'individus en se basant sur les sept échelles de confort définies par ASHRAE, en s'étalant de -3 (très froid) à +3 (très chaud). A l'origine, l'indice du PMV était 
formulé pour estimer le confort thermique intérieur, mais comme sa valeur a une relation avec l'équilibre d'énergie, elle pourra aussi atteindre des valeurs plus élevées ou plus basses, cependant, il a été récemment appliqué pour les conditions extérieures aussi.

Ce paramètre est calculé en résolvant l'équation modifiée de Fanger (Fanger 1972) [26] développée par Jendritzky et Nübler (1981) [27] pour les conditions de confort thermique extérieur.

L'équation d'équilibre énergétique décrivant l'échange de chaleur entre le corps humain et l'environnement thermique est la suivante:

$M+W+Q(\mathrm{MRT}, v)+Q_{H}(\mathrm{Ta}, v)+Q_{L}(e, v)+Q_{S W}(e, v)+Q_{R e}(\mathrm{Ta}, e)=S$

Avec :

M : le taux de métabolisme

$\mathrm{W}$ : le rendement du travail physique

Q : le bilan radiatif

MRT : température moyenne radiante

$\mathrm{V}$ : vitesse de l'air

$\mathrm{QH}$ : le flux de chaleur turbulente de la chaleur sensible

Ta : température de l'air

QL : le flux de chaleur latente due à l'évaporation de l'humidité diffuse à travers la peau

e : humidité de l'air

QSW : le flux de chaleur latente d'évaporation de la sueur

QRe : le flux de chaleur respiratoire (sensible et latente)

$\mathrm{S}:$ le stockage

Tous les termes de l'équation. (1) sont en [w/m²]. Ces termes de cette équation ont des signes positifs s'ils gagnent de l'énergie pour le corps (M est toujours positif, W, QL et Q SW sont toujours négatifs).

Michael Bruse dans ses travaux effectués lors de l'actualisation de son programme de simulation (ENVI-met) [26] a adopté neuf échelles de confort propres aux espaces extérieurs 
de -4 (extrêmement froid) à +4 (extrêmement chaud).

Pour notre cas d'étude, l'estimation du confort thermique extérieur par l'indice du PMV déduit par l'exploitation du logiciel Envi-met 3.1 a donné ce qui suit :

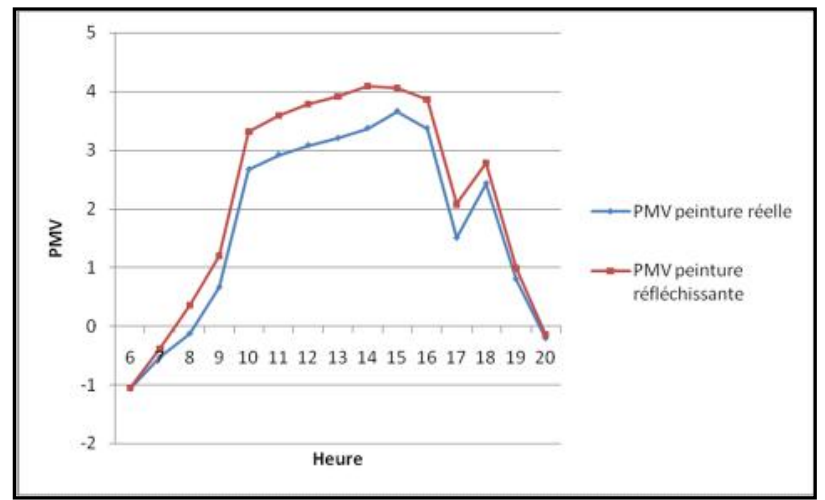

Fig.7. Variation du PMV pour les peintures réelles et les peintures réfléchissantes

La période de $10 \mathrm{~h}$ du matin à $18 \mathrm{~h}$ marque une nette distinction entre les valeurs des deux peintures simulées, en enregistrant des valeurs supérieures pour les peintures réfléchissantes comparées à celles des peintures standards.

A partir de 10h, les valeurs du PMV dépassent déjà la valeur 2 qui exprime la sensation de «chaud», avec un écart de 0.65 plus élevé pour les peintures réfléchissantes que celles standards, et cette allure continue jusqu'à 16h, en enregistrant des valeurs qui varient entre 3 et 4 exprimant des sensations de «très chaud» à « extrêmement chaud», toujours avec des valeurs plus élevées pour les peintures réfléchissantes que pour les peintures standards, en ayant comme maximum d'écart une valeur de 0.72 à $14 \mathrm{~h}$. Pendant cette période, l'espace reçoit directement le rayonnement solaire qui sera plus réfléchi par les surfaces peintes en clair (réfléchissantes) que les peintures standards, ce qui soumettra les piétons à une multitude de réflexions du sol et des façades environnantes, ce qui permettra de passer dans l'échelle de ASHRAE de la sensation «chaud » à «très chaud » et « extrêmement chaud », et donc, avoir un sentiment d'inconfort et de stress thermique.

Entre $17 \mathrm{~h}$ et $18 \mathrm{~h}$, il y a eu une baisse de la valeur du PMV, qui s'approchera de la valeur 2 , c'est-à-dire sensation de «chaud», et puis augmente de nouveau, pour s'approcher de 3 «très chaud », ce balancement est du à l'absence du soleil dans l'espace à $17 \mathrm{~h}$, car il est obstrué par un bâtiment voisin, puis son exposition de nouveau au rayonnement direct à $18 \mathrm{~h}$, 
lors de sa déviation vers l'ouest avant son coucher.

Cela coïncide avec plusieurs études anciennes et récentes, qui mettent en évidence les effets négatifs des couleurs claires réfléchissantes sur le confort thermique extérieur ainsi que la dégradation du confort visuel. A titre indicatif, Ghaffarianhoseini et al.2015 [24] qui ont trouvé que l'augmentation de l'albédo des murs d'enceintes dans des cours urbaines réduit considérablement le confort thermique extérieur, et que le niveau le plus élevé du PMV appartient à la cour du plus haut albédo, la valeur du PMV de la cour avec un albédo de 0,3 est d'environ 4 indiquant un malaise, tandis qu'un albédo de 0,55 se traduit par une augmentation de la valeur PMV à 5, alors qu'un albédo de 0,93 conduit à une valeur de PMV de 6.

De même pour les études de Taleghani et al.2014 [22], Mansouri et al.2008 [28], Nikolopoulou et al.2002 [29] et Chatzidimitriou et al.2006 [7], qui témoignent toutes sur l'effet opposé (rapport inversement proportionnel) de l'augmentation de l'albédo sur le confort thermique extérieur.

\subsection{Température Physiologique Equivalente}

Afin d'estimer le confort thermique extérieur par le calcul de la température physiologique équivalente (PET), on a eu recours au logiciel Rayman 1.2, développé par Matzarakis et al [30] et[31].

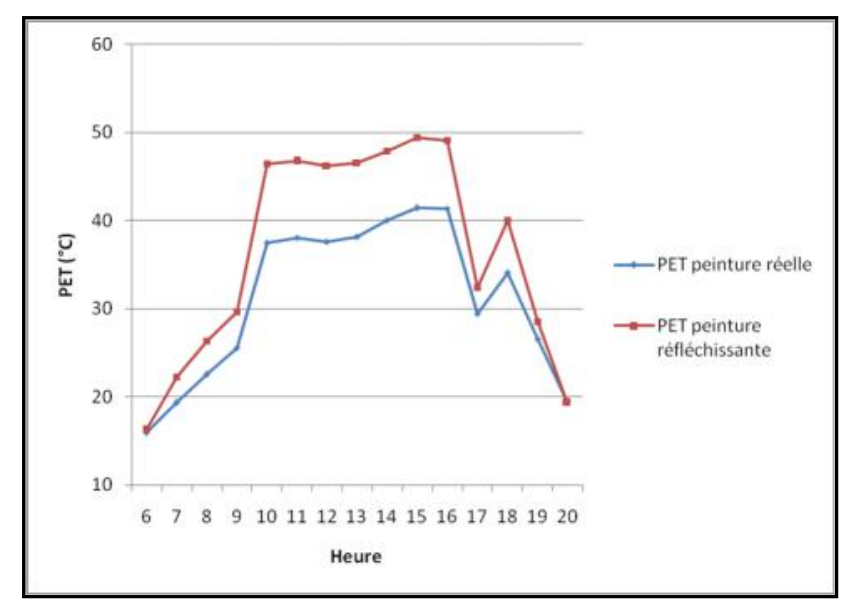

Fig.8. Variation de la PET pour les peintures réelles et les peintures réfléchissantes

La température physiologique équivalente (PET) marque des fluctuations proportionnelles à celles de la Tmrt, elle prend aussi la même allure que les variations du PMV. 
Du lever au coucher du soleil, les valeurs de la PET relatives à la peinture réfléchissante sont plus élevées que celles de la peinture standard, en marquant une valeur maximale de $49.4^{\circ} \mathrm{C}$ à $15 \mathrm{~h}$ pour la peinture réfléchissante contre une température de $41.4^{\circ} \mathrm{C}$ pour la peinture standard d'où un écart de $8^{\circ} \mathrm{C}$. L'écart maximal qui est de $8.9^{\circ} \mathrm{C}$ est marqué à $10 \mathrm{~h}$.

Ces résultats montrent que la période d'excès de chaleur (période où l'intensité du rayonnement solaire est la plus forte), exprime une valeur très élevée de la PET (extrêmement chaud), ce qui se traduit par une forte sensation d'inconfort, et on la remarque plus accentuée pour la peinture réfléchissante que pour la peinture standard.

Nos résultats coïncident avec ceux de Ghaffarianhoseini et al.2015 [24], qui ont trouvé que les valeurs de la PET calculée montrent que l'augmentation des valeurs d'albédo des surfaces des parois se traduit par une réduction significative des conditions de confort thermique. Ils disent qu'il est évident que les valeurs de la PET soient au-dessus du plus large éventail de confort $\left(22-34^{\circ} \mathrm{C}\right)$ dans toutes les cours pendant la période critique de $12 \mathrm{~h}-15 \mathrm{~h}$, tandis qu'une meilleure performance thermique des cours avec un albédo plus faible peut être appréciée.

Dans une autre étude dans le climat subtropical de Shanghai, Yang et al. (2011) [32] ont montré qu'en augmentant l'albédo de surface du sol de 0,4 , le confort thermique extérieur global a diminué, comme en témoigne une augmentation dans la température physiologique équivalente (PET) de 5 à $7^{\circ} \mathrm{C}$.

\subsection{Corrélation entre le PMV et la PET}

D'après les deux graphes ci-dessous, nous remarquons qu'il y a une forte corrélation entre les valeurs du PMV et celles de la PET, que ce soit pour la peinture réelle (standard) ou la peinture réfléchissante (cool), avec un $\mathrm{R}^{2}=0.99$ et 0.98 respectivement, qui est une valeur assez élevée et qui accentue les résultats obtenus pour l'estimation du confort thermique extérieur par le biais des deux paramètres (PMV et PET).

Donc, le confort thermique au niveau des espaces extérieur se dégrade en augmentant la valeur d'albédo, ce qui fait que le piéton soumis aux multitudes réflexions dues aux surfaces réfléchissantes qui l'entourent (surfaces peintes en clair), se sentira à l'inconfort. 

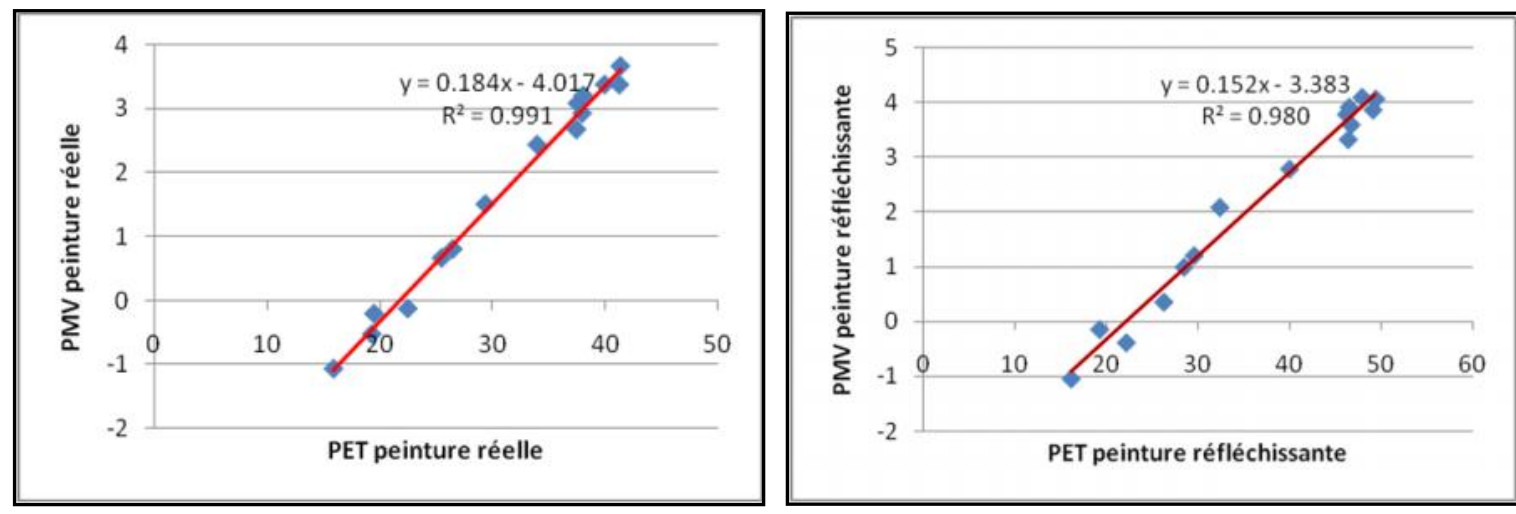

Fig.9. La corrélation entre les valeurs du PMV et de la PET pour la peinture réelle et la peinture réfléchissante

\section{CONCLUSION}

L'étude de l'effet de l'albédo sur le confort thermique extérieur, nous a permis par le biais de cette étude basée sur des simulations numériques de déduire une très bonne corrélation entre les deux paramètres choisis pour l'estimation du confort thermique dans notre aire d'étude et qui sont le PMV estimé par Envi-met3.1 et la PET estimée par Rayman1.2. Les résultats obtenus révèlent que les valeurs les plus élevées relatives à une sensation d'inconfort "chaud" à "très chaud" et même "extrêmement chaud" correspondent aux surfaces avec peintures claires réfléchissantes pendant la période de la journée où le rayonnement solaire est le plus intense, malgré que ces peintures ont suscité une modération du microclimat par l'atténuation des températures de surfaces du sol et de l'air. Donc, on peut conclure que contrairement à ce que peuvent avoir des matériaux à haut albédo comme effet sur l'atténuation de l'effet d'îlot de chaleur urbain, la modération du microclimat et la diminution de la consommation énergétique, il donne un effet inverse concernant la sensation de confort que pourra sentir un être humain debout (piéton) au niveau d'un espace extérieur. Cela coïncide avec plusieurs recherches au niveau de la climatologie urbaine et de l'environnement à travers le monde. Cependant, le fait que les surfaces colorées en clair (réfléchissantes) peuvent augmenter la quantité des radiations atteignant un corps exposé, soulève les questions à propos de leur convenance pour leur utilisation dans les espaces extérieurs urbains, surtout concernant les conditions de confort des piétons. Ce qui ouvre des perspectives et donne à réfléchir à de nouveaux axes de recherches, tel que le fait de tester la texture du matéraiux (chose qui n'est 
pas possible par Envi-met), et voir l'effet de la couleur claire mais non réfléchissante sur le confort thermique des usagers des espaces extérieurs.

\section{REFERENCES}

[1] Onu "World urbanisation prospects", Progress in planning, 2011, AIE \& PNUE in J-J.

Terrin, Villes et changement climatique. îlots de chaleur urbains. Marseille: Editions Parenthèses, 2015,pp.11.

[2] Pigeon G, Lemonsu A, Masson V, Hidalgo J. De l'observation du microclimat urbain à la modélisation intégrée de la ville. La Mééorologie, 2008, 62, 39.

[3] Taha H, Akbari H, Rosenfeld A, Huang J. Residential Cooling Loads and The Urban Heat Island-the effects of Albedo, Building and environment, 1988, 23 (4): 271-283.

[4] Taha H, Sailor D, Akbari H. High albedo materials for reducing cooling energy use. Lawrence Berkeley Lab. Rep.31721 UC-350. Berkeley, CA, 1992.

[5] Taha H. Analysis of Energy Efficiency of Air Quality in the South Coast Air Basin- Phase II. Lawrence Berkeley National Laboratory Report N.LBL-35728, Berkeley, CA, 1994: Ch6:161-218.

[6] Rosenfeld A H, Akbari H, Bretz S, Fishman B L, Kurn D M, Sailor D, Taha H. Mitigation of urban heat island materials unity programs, and updates, Energy and Building 1995, 22: 255-265.

[7] Chatzidimitriou A, Chrissomallidou N, Yannas S. Ground surface materials and microclimates in urban open spaces. PLEA, The 23 rd conference, Geneva, Switzerland, 2006.

[8] Shiraishi Y et al, 2005 in Chatzidimitriou A, Chrissomallidou N, Yannas S. Ground surface materials and microclimates in urban open spaces. PLEA, The 23 rd conference, Geneva, Switzerland, 2006.

[9] Santamouris M. The role of materials. Impact on the temperature regime of cities, Ec-Asean Energy Facility (EAEF), Singapore, 2006.

[10] Akbari H, Bretz S, Kurn D M, Hanford J. Peak power and cooling energy savings of high-albedo roofs, Energy and building, 1997, 25: 117-126.

[11] Prado R T A, Ferreira F L. Measurement of albedo and analysis of its influence the surface temperature of building roof materials, Energy and building, 2005, 35: p.295-300. 
doi:10.1016/j.enbuild.2004.03.009

[12] Capderou M. Atlas solaire de l'Algérie. Tome2, Aspect énergétique, 1985, pp12.

[13] Station météorologique de Skikda.

[14] Reagan J A, Acklam D M. Solar Reflectivity of Common Building Materials and its Influence on the Roof Heat Gain of Typical Southwester U.S.A. Residences. Energy and building, 1979, 2: 237-248.

[15] http://eetd.lbl.gov/HeatIsland/Pavements/Lowertemperatures/

[16] Synnefa A， Santamouris M, Akbari H. Estimating the effect of using cool coatings on enrgy loads and thermal comfort in residential buildings in various climatic conditions, Energy and Buildings, 2007, 39: 1167-1174. doi:10.1016/j.enbuild.2007.01.004

[17] Doulos L, Santamouris M, Livada I. Passive cooling of outdoor urban spaces. The role of materials. Solar Energy, 2004, 77: 231- 249. doi:10.1016/j.solener.2004.04.005

[18] Priyadarsini R, Wong N H. Building surfaces and their effect on the urban thermal environment, Architectural science review, 2005, 48(4): 345-356.

[19] ISO 7729, Ergonomics of the Thermal Environment - Instruments for measuring Physical Quantities. International Standardisation Organisation, Geneva.1998.

[20] Ali-Toudert F, Mayer H. Numerical study on the effects of aspect ratio and orientation of an urban street canyon on autdoor thermal comfort in hot and dry climate, Building and envirpnment, 2006, 14: 94-108.

[21] Halawa E, Hoof J V, Soebarto V. The impacts of the thermal radiation field on thermal comfort energy consumption and control- A critical overview, Renewable and sustainable Energy Reviews, 2014, 37: 907-918. doi.org/10.1016/j.rser.2014.05.040

[22] M. Taleghani, M.Tenpierik, A.V.D. Dobbelsteen, D.J.Sailor, Heat in courtyards : A validated and calibrated parametric study of heat mitigation strategies for urban courtyards in the Netherlands, Solar Energy, 2014, 103: 108-124. doi.org/10.1016/j.solener.2014.01.033

[23] Taleghani M, Sailor D J, Tenpierik M, Dobbelsteen A V D. Thermal assessment of heat mitigation strategies: the case of Portland State University, Oregon, USA, Build. Environ, 2014, 73: 138-150.

[24] Ghaffarianhoseini A, Berardi U, Ghaffarianhoseini A. Thermal performance characteristics of unshaded courtyards in hot and humid climates, Building and Environment, 2015, 87: 154-168. doi.org/10.1016/j.buildenv.2015.02.001

[25] Fanger P O. Thermal comfort, 1972. in. Berkovic S, Yezioro A, Bitan A. Study of thermal comfort in courtyards in a hot arid climate, Solar Energy, 2012, 86: 1173-1186. 
doi:10.1016/j.solener.2012.01.010

[26] Jendritzky G., Nübler W. in Berkovic S, Yezioro A, Bitan A. Study of thermal comfort in courtyards in a hot arid climate, Solar Energy, 2012, 86: 1173-1186. doi:10.1016/j.solener.2012.01.010

[27] Bruse M. ENVI-met 3.1 Disponible depuis : http://www.envi- met.com.

[28] Mansouri O. L’influence de la réflectivité des matériaux (albédo) sur la modification du microclimat et sur le confort thermique extérieur dans un canyon urbain, mémoire de Magister, université Mentouri. Contantine.2008.

[29] Nikolopoulou M. in RUROS, Rediscovering the Urban Realm and Open Spaces, 2002, pp 46.

[30] Matzarakis A, Rutz F, Mayer H. Modelling radiation fluxes in simple and complex environments - Application of the RayMan model. Int J Biometeorol, 2007, 51: 323-334. DOI 10.1007/s00484-006-0061-8

[31] Matzarakis A, Rutz F, Mayer H. Modelling radiation fluxes in simple and complex environments: basics of the RayMan model. Int J Biometeorol, 2010, 54:131-139. DOI 10.1007/s00484-009-0261-0

[32] Yang et al.(2011). in Taleghani M, Tenpierik M, Dobbelsteen A V D, Sailor D J. Heat in courtyards: A validated and calibrated parametric study of heat mitigation strategies for urban courtyards in the Netherlands, Solar Energy, 2014, 103: 108-124. doi.org/10.1016/j.solener.2014.01.033

How to cite this article:

Mansouri O, Bourbia F, Belarbi R. Effect of urban albedo surfaces on thermal comfort. J. Fundam. Appl. Sci., 2017, 9(2), 878-896. 